\title{
GASTROSTOMIA DE INCISÃO ÚNICA COMO ALTERNATIVA PARA O PROCEDIMENTO ENDOSCÓPICO
}

\author{
SINGLE-WOUND GASTROSTOMY: A SIMPLE METHOD AS AN OPTION FOR \\ ENDOSCOPY
}

\author{
Ricardo Zorrón, TCBC-RJ'1; Daniel Flores²; Carlos André Fontes Meyer²; \\ Leonardo Machado de Castro, AsCBC-RJ²; Fábio Athayde Veloso Madureira, AsCBC-RJ'2; \\ Delta Madureira Filho, TCBC-RJ ${ }^{3}$
}

\begin{abstract}
RESUMO: Objetivos: A gastrostomia, realizada para possibilitar acesso nutricional ou descompressão, é comumente realizada por via endoscópica, por radiologia intervencionista, e, mais raramente, por via cirúrgica através de videolaparoscopia ou incisão mediana supra-umbilical. Os métodos endoscópico e radiológico, apesar de estabelecidos em muitos centros, não constituem rotina em hospitais comunitários pela necessidade de pessoal qualificado e material descartável, sendo usualmente realizada a gastrostomia cirúrgica com incisão mediana e anestesia geral. A Gastrostomia de Incisão Única descrita neste trabalho é um novo método cirúrgico menos invasivo, que foi desenvolvido para ser realizado sob anestesia local, com uma única incisão e sem necessidade de equipamento especial. Método: Quinze pacientes elegíveis para gastrostomia por diferentes indicações foram operados sob anestesia local. Após incisão subcostal de $1 \mathrm{~cm}$ sobre o músculo reto abdominal esquerdo, uma área de parede gástrica era localizada, com ajuda de azul de metileno injetado no estômago, e exteriorizada através da incisão. Duas suturas em bolsa eram realizadas e a sonda gástrica introduzida. O estômago era fixado à aponeurose, resultando em uma gastrostomia de incisão única, segura e bem fixada. Alimentação pôde ser iniciada através da gastrostomia em 24 a 48hs. Resultados: Todos os pacientes foram submetidos à técnica com sucesso. Complicações menores ocorreram em três pacientes: um apresentou alargamento do estoma, necessitando de sutura local adicional, e em outro, ocorreu deiscência parcial de mucosa que foi tratada conservadoramente. Em um terceiro paciente, houve lesão iatrogênica de cólon transverso, que foi suturada sem intercorrências. O tempo operatório médio foi de 49,4 min, e a introdução de alimentação foi de 34,3 hs em média. Não houve falha na instituição de dieta com esta técnica, nem complicações maiores como fístula ou peritonite. Conclusão: Os resultados desta casuística inicial sugerem que a abordagem é efetiva em propiciar um acesso rápido e seguro para gastrostomia, com ferida única, dispensando o uso de suporte endoscópico e anestesia geral. Pode ser utilizado como opção ao método endoscópico em pacientes com obstrução neoplásica faríngea ou esofageana. Uma casuística mais expressiva, acompanhamento mais longo dos casos, e estudos prospectivos randomizados são necessários para estabelecer o papel desta nova técnica na rotina cirúrgica (Rev. Col. Bras. Cir. 2005; 32(3): 153-156).
\end{abstract}

Descritores: Gastrostomia/ instrumentação/ métodos; Procedimentos cirúrgicos do sistema digestório; Estudos prospectivos/ Rio de Janeiro.

\section{INTRODUÇÃO}

A gastrostomia é uma das cirurgias abdominais mais antigas, realizada desde o início do século XIX. Variadas técnicas cirúrgicas têm sido descritas e utilizadas desde então, mas a abordagem cirúrgica vem perdendo espaço por técnicas menos invasivas ${ }^{1-4}$. A gastrostomia para descompressão ou para acesso nutricional é rotineiramente realizada por via endoscópica, mais recentemente por radiologia intervencionista por punção, e mais raramente por via cirúrgica, através de videolaparoscopia ou por incisão mediana, usualmente sob anestesia geral ${ }^{5,6}$. Embora possa ser realizada com segurança razoável, variados graus de dificuldade técnica e complicações vêm sendo descritos. Hemorragia, descolamento do estômago da parede abdominal, instalação do tubo no cólon transverso, e fístula com peritonite,podem ser encontrados em 13 a $23 \%$ com os métodos cirúrgicos atuais, em 10 a 20\% com o método endoscópico, e relatado como significativamente menor para gastrostomia sob controle radiológico $(1,6 \text { a } 6 \%)^{7-12}$. Além disso, pacientes com neoplasia obstrutiva ou estenose esofageana não são candidatos para o método endoscópico. Para pacientes com indicação de gastrostomia cirúrgica, é desejável evitar uma incisão mediana e anestesia geral, mas pouco viável com os métodos atuais.

Este trabalho descreve uma nova técnica desenvolvida pelo autor para gastrostomia cirúrgica descompressiva ou com fins de nutrição, a Gastrostomia Puntiforme, que utiliza uma pequena e única incisão, sob anestesia local e sem uso de suporte endoscópico.

\section{MÉTODO}

Entre julho de 2003 e fevereiro de 2004, quinze pacientes foram submetidos ao método. O único critério de exclu-

1. Cirurgião do Serviço de Cirurgia - Hospital Universitário Clementino Fraga Filho (H.U.C.F.F.-U.F.R.J.); Coordenador do Centro de Cirurgia Minimamente Invasiva - Hospital Municipal Lourenço Jorge - RJ; Regente da Cadeira de Cirurgia - Faculdade de Medicina de Teresópolis FESO; Membro Titular da Sociedade de Cirurgia Vídeo-endoscópica do Rio de Janeiro (SOCIVERJ).

2. Cirurgião Residente do Serviço de Cirurgia - Hospital Universitário Clementino Fraga Filho (H.U.C.F.F.-U.F.R.J.).

3. Professor Adjunto do Departamento de Cirurgia da Faculdade de Medicina da UFRJ; Chefe do Serviço de Cirurgia - Hospital Universitário Clementino Fraga Filho (H.U.C.F.F.-U.F.R.J.); Membro Titular da Sociedade de Cirurgia Vídeo-endoscópica do Rio de Janeiro (SOCIVERJ).

Recebido em 03/03/2005

Aceito para publicação em 10/05/2005

Trabalho realizado no Serviço de Cirurgia do Hospital Universitário Clementino Fraga Filho (H.U.C.F.F.-U.F.R.J.) - Rio de Janeiro. 


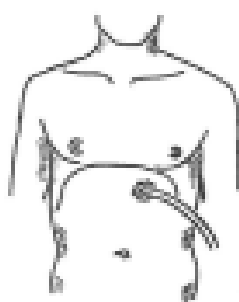

A

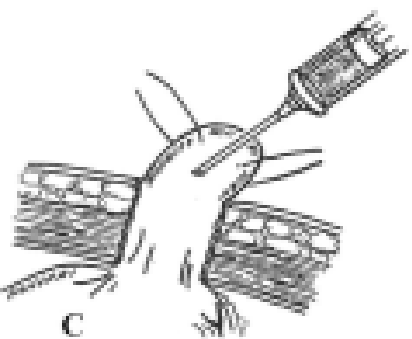

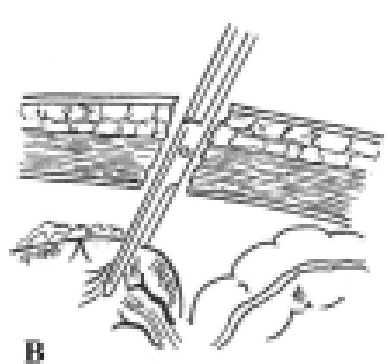

B

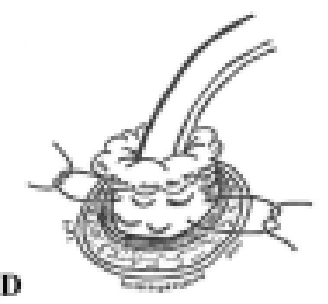

Figura 1 - A. Localização esperada para Gastrostomia Puntiforme. B. Busca da parede gástrica após insuflação com ar. C. Aspiração de azul de metileno confirma conteúdo gástrico. D. Fixação à aponeurose e pele, realizando uma dupla sutura em bolsa.

são foi para pacientes com índice de massa corporal (IMC) acima de $32 \mathrm{~kg} / \mathrm{m} 2$, os quais foram preferencialmente submetidos a outras técnicas. Dados operatórios e de acompanhamento pós-operatório foram prospectivamente documentados. As indicações para gastrostomia, bem como dados demográficos da amostra do estudo são listados na Tabela 1. A idade variou de 17 a 87 anos, com uma média de 58,7 anos.

O paciente era posicionado em decúbito dorsal, e um ponto no meio do músculo reto abdominal esquerdo, distando $3 \mathrm{~cm}$ abaixo do rebordo costal, é escolhido para a incisão (Figura1A). Uma pequena incisão transversa de $1 \mathrm{~cm}$ é realizada sob infiltração local com $10 \mathrm{ml}$ de marcaína a 0,5\%, da pele ao peritôneo. Sedação leve com midazolam $(0,15$ a $0,3 \mathrm{mg} /$ $\mathrm{kg})$ e propofol $(1 \mathrm{mg} / \mathrm{kg})$ é realizada previamente à infiltração. Suturas com fio 2.0 são instaladas fixando cada lado das bordas peritoneais e tracionados para melhor exposição. $\mathrm{O}$ estômago é insuflado por ar pelo anestesiologista através da sonda naso-gástrica, quando possível sua colocação. Uma pinça é introduzida na incisão e inicia-se uma procura por parede gástrica anterior, que é facilmente visível após a insuflação (Figura 1B). Usualmente o órgão obtido pode ser parcialmente exteriorizado e observado seu aspecto "gástrico"ou “colônico". Se o segmento obtido parece inadequado, ele é reintroduzido na cavidade e uma nova busca é iniciada a partir da mesma incisão. Com o segmento gástrico adequado tracionado pela incisão, $100 \mathrm{ml}$ de solução de azul de metileno são instilados através da sonda nasogástrica (quando possível a passagem da sonda), e o conteúdo gástrico é puncionado para confirmar a presença de azul, confirmando o posicionamento correto da gastrostomia (Figura 1C). Se a instalação de sonda nasogástrica não é possível ou contraindicada (devido a obstrução faringo-esofageana por exemplo), então $50 \mathrm{ml}$ de contraste iodado são instilados na alça exteriorizada, e uma radiografia panorâmica é obtida para confirmar o posicionamento correto (Figura 2).
Com a confirmação do conteúdo gástrico, são realizadas duas camadas concêntricas de sutura em bolsa de Prolene 2.0, que constituirão uma válvula da parede do estômago, que é fixada à aponeurose anterior do reto abdominal utilizando as mesmas suturas (Figura 1D). Uma sonda de gastrostomia ou Folley diâmetro 18 a 22 é inserida e o balão é inflado com $5 \mathrm{ml}$ de solução fisiológica. A sonda é tracionada e suturada com fixação à pele com Mononylon 2.0 (Figura 3).

\section{RESULTADOS}

A técnica foi empregada com sucesso em todos os pacientes, e não houve necessidade de expansão da incisão ou de realização de nova incisão. O teste de azul de metileno confirmou o correto posicionamento da agulha dentro do estômago em todos os pacientes, com exceção de dois pacientes com obstrução esofageana, nos quais foi utilizado controle radiológico. A apreensão do estômago foi conseguida por visão direta do órgão expandido pela insuflação por vezes com auxílio de afastador dentro da incisão. Raramente ocorreu dúvida quanto ao órgão exteriorizado pela incisão, e nestes casos, o controle radiológico ou a aspiração do azul de

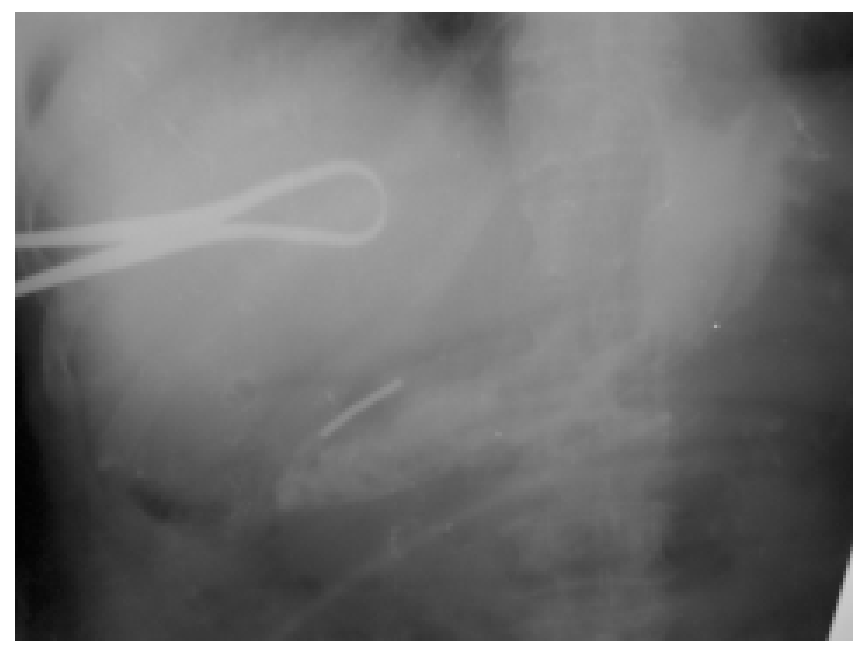

Figura 2 - Controle radiológico intra-operatório obtido após punção e instilação de contraste iodado no lumen gástrico.

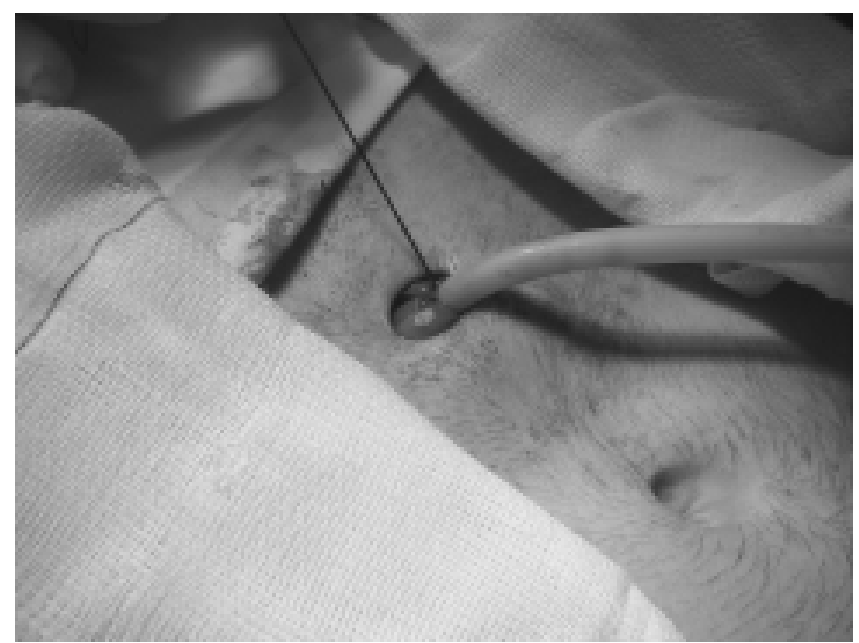

Figura 3 - Aspecto operatório da Gastrostomia Puntiforme. 
Tabela 1 - Indicações e Dados demográficos da população do estudo.

\begin{tabular}{lcclcccl}
\hline & Idade & Sexo & \multicolumn{1}{c}{ Indicação } & $\begin{array}{c}\text { Tempo } \\
\text { Op.(min) }\end{array}$ & $\begin{array}{c}\text { Perda } \\
\text { Sangue } \\
\text { (ml) }\end{array}$ & $\begin{array}{c}\text { Nutrição } \\
\text { pela } \\
\text { sonda (hs) }\end{array}$ & Complicações \\
\hline Paciente 1 & 68 & M & Neoplasia esofageana & 46 & 5 & 48 & - \\
Paciente 2 & 70 & F & AVE+seqüela & 35 & 15 & 24 & - \\
Paciente 3 & 75 & F & AVE +seqüela & 35 & 10 & 24 & - \\
Paciente 4 & 76 & F & AVE + seqüela & 35 & 12 & 24 & Deiscência de mucosa \\
Paciente 5 & 19 & M & SIDA + Candidíase esofageana & 20 & 15 & 24 & - \\
Paciente 6 & 21 & M & Traumatismo Crânio-encefálico & 38 & 5 & 24 & Alargameto de estoma \\
Paciente 7 & 81 & F & AVE + seqüela & 54 & 25 & 48 & - \\
Paciente 8 & 58 & F & Carcinoma de Orofaringe & 40 & 20 & 48 & - \\
Paciente 9 & 74 & F & Neoplasia esofageana & 60 & 40 & 48 & - \\
Paciente 10 & 17 & M & Paralisia cerebral & 100 & 100 & 48 & Laceração de cólon \\
Paciente 11 & 67 & M & Neoplasia esofageana & 45 & 20 & 24 & - \\
Paciente 12 & 74 & M & AVE + seqüela & 50 & 5 & - & s/acompanha-mento \\
Paciente 13 & 23 & F & Tu. Oral - Linfoma angiocêntrico & 50 & 50 & 48 & - \\
Paciente 14 & 71 & M & AVE +seqüela & 60 & 20 & 24 & - \\
Paciente 15 & 87 & F & Demência multivascular & 72 & 20 & 24 & - \\
Média & & & & 49,4 & 24,1 & 34,3 & 3 (20\%) \\
\hline
\end{tabular}

metileno foi suficiente para confirmar a alça exteriorizada. Em um paciente,no entanto, ocorreu lesão de cólon transverso de $5 \mathrm{~mm}$, que foi suturada e evoluiu sem intercorrências. Neste paciente, uma gastrostomia tradicional a Stamm foi realizada pela incisão. O tempo operatório foi de 49,4 min em média, variando de 20 a 100min (Tabela 1). Perda sangüínea estimada média foi de 24,1 $\mathrm{ml}$ (variando de 5 a 100ml). Efetiva nutrição pela gastrostomia foi iniciada em 24 a 48 hs após o procedimento (média de 34,3 hs).

O tempo de acompanhamento foi de duas a quatro semanas, em virtude de a maioria dos pacientes receberem alta para tratamento em regime de home care, não sendo esperado retorno para consulta ambulatorial. Resultados da técnica a longo prazo, portanto, ainda não são conhecidos, mas a evolução precoce sugere ser similar à das técnicas existentes. Além da perfuração do cólon, complicações menores ocorreram em dois pacientes: em um houve vazamento excessivo do conteúdo gástrico em volta da sonda que necessitou de sutura adicional com fio inabsorvível para estreitar o orifício. O segundo apresentou deiscência parcial da mucosa do estômago que foi tratada com cuidados locais, sendo que a nutrição pela sonda não foi interrompida.

\section{DISCUSSÃo}

A endoscopia e a radiologia invasiva modificaram a abordagem cirúrgica, estabelecendo soluções relativamente simples e menos invasivas para a gastrostomia. Evitando uma incisão mediana e anestesia geral utilizada na maioria das técnicas mais consagradas, seu uso, entretanto, pode resultar desapontador para hospitais de comunidade, onde podem não existir profissionais habilitados para endoscopia e radiologia intervencionista, além do custo ser elevado para o material necessário. Complicações também vêm sendo descritas, com perigo de morbidade mais grave como perfuração de cólon e intestino delgado, hemorragia intra-abdominal, fasciíte e peritonite, estando estes métodos relacionados com uma mortalidade em torno de $0,6 \% \%^{7-9,13,14}$. Outras complicações variadas como fístula gastro-cólica, invaginação, migração, obstrução e nutrição intra-parietal inadvertida vêm sendo descri$\operatorname{tas}{ }^{15-18}$.

A técnica de Gastrostomia por Incisão Única desenvolvida em nossa instituição, é uma conseqüência lógica das abordagens minimamente invasivas que vêm revolucionando a terapia cirúrgica em todas as especialidades ${ }^{10,19}$. Com a particularidade de dispensar tecnologia avançada, e poder ser utilizada em qualquer unidade hospitalar com centro cirúrgico, este método de simples aprendizado pode ser aplicado na maioria dos pacientes elegíveis para gastrostomia de propósito nutricional ou descompressivo. Vantagens do método são o fácil acesso sob anestesia local, menor trauma tecidual, ausência de auxílio endoscópico e na maioria das vezes, de controle radiológico, e curva de aprendizado abreviada para programas de treinamento. Como a cavidade não é aberta por via mediana, não deve ocorrer íleo pós-operatório, nem aderências relacionadas às incisões maiores. A ocorrência de vazamento do conteúdo gástrico na cavidade peritoneal, relatada para todas as outras técnicas, não é esperada para esta gastrostomia, uma vez que a parede gástrica é fixada na aponeurose anterior do reto abdominal, e não internamente ao peritôneo como nas técnicas conhecidas, inclusive na gastrostomia endoscópica e por radiologia intervencionista.

Limitações do método são pacientes obesos (IMC > $32 \mathrm{~kg} / \mathrm{m} 2$ ), pela dificuldade de localização e tração excessiva do órgão exteriorizado, e pacientes com retração gástrica por atrofia. As indicações desta abordagem e comparação com o método endoscópico estão sendo avaliadas por um estudo prospectivo randomizado em nossa instituição. 
Os resultados desta casuística inicial sugerem que a abordagem é efetiva em propiciar um acesso rápido e seguro para gastrostomia, com ferida única, dispensando o uso de suporte endoscópico e anestesia geral. A simplicidade e potenciais vantagens do método podem ser obser- vadas nos resultados desta casuística inicial, mas um acompanhamento mais longo, um grupo de pacientes mais expressivo, e estudos prospectivos randomizados são necessários para estabelecer o papel desta técnica menos invasiva.

\begin{abstract}
Background: Gastrostomy for feeding or descompressive purposes are often performed transendocopically, by fluoroscopic guidance, and more rarely, by laparoscopy or by an abdominal midline incision. As endoscopic and radiologic personel and instrumental are often not wide available in community hospitals in our country, an alternative method was developed in our institution. A single-wound, surgical access gastrostomy, performed with local anesthesia and no need for endoscopic guidance, is described. Methods: Fifteen patients elegible for gastrostomy were submitted to the technique. The patients were managed under local anesthesia, and after a $1 \mathrm{~cm}$ incision on the left rectus abdominal muscle, an area of gastric wall is localized under Methylene Blue testing. With confirmation of gastric content, two layers of concentric purse string unabsorbable sutures are placed to work as a valve, and the stomach is fixed to the rectus sheath, resulting in a safe single wound gastrostomy. The feeding through the tube was started in 24 to 48hs. Results: All patients were successfully managed by this method. Minor complications ocurred in 3 patients: one enlargement of the gastrostomy required additional suture, and one partial mucosal dehiscence was clinically managed. In a third patient, an iatrogenic transverse colon laceration ocurred, and suture closure was performed without further morbidity. Mean operative time was 49.4 min, mean institution of gastrostomy feeding was 34.3 hs. There was no failure to achieve postoperative feeding nor major complications as leakage or peritonitis. Conclusion: Our results suggest that this new approach is effective and safe, providing a single wound access, avoiding the need for endoscopic guidance and general anesthesia. It can also be used instead of the endoscopic method in patients with faringo-esophageal obstruction. A larger number of cases, longer follow-up observation and prospective randomized studies are needed to establish the role of the new technique in a routine basis.
\end{abstract}

Key-words: Gastrostomy/instrumentation/methods; Digestive system surgical procedures; Prospective studies/Rio de Janeiro.

\section{REFERÊNCIAS}

1. Stamm M. Gastrostomy: a new method. Med News. 1894;65:324

2. Witzel O. Zur Technik der Magenfistulaeinlegung. Zbl Chir. 1891;18:601-4.

3. Spivack JL. Eine neue Methode der Gastrostomie. Beitr Klin Chir. 1929;147:308.

4. Moss G. A simple technique for permanent gastrostomy. Surgery. 1972;71(3):369-70.

5. Reiner DS, Leitman IM, Ward RJ. Laparoscopic Stamm gastrostomy with gastropexy. Surg Laparosc Endosc. 1991;1(3):189-92.

6. Lathrop JC, Felix EJ, Lauber D. Laparoscopic Janeway gastrostomy utilizing an endoscopic stapling device. J Laparoendosc Surg. 1991;1(6):335-9.

7. Stiegman G, Goff J, Van Way C, et al. Operative versus endoscopic gastrostomy. Preliminary results of a prospective randomized trial. Am J Surg. 1988;155(1):88-92.

8. O'Keeffe F, Carrasco CH, Charnsangavej C. Percutaneous drainage and feeding gastrostomies in 100 patients. Radiology. 1989;172(2):341-3.

9. Halkier BK, Ho CS, Yee AC. Percutaneous feeding gastrostomy with the Seldinger technique: review of 252 patients. Radiology.1989;171(2):359-62.

10. Hicks ME, Surratt RS, Picus D, et al. Fluoroscopically guided percutaneous gastrostomy and gastroenterostomy: analysis of 158 consecutive cases. AJR Am J Roentgenol. 1990;154(4): 725-8.

11. Saini S, Mueller PR, Gaa J, et al. Percutaneous gastrostomy with gastropexy: experience in 125 patients. AJR Am J Roentgenol.1990;154(4):1003-6.
12. Deutsch LS, Kannegieter L, Vanson DT, et al. Simplified percutaneous gastrostomy. Radiology. 1992;184(1):181-3.

13. Ponsky JL, Gauderer MW. Percutaneous endoscopic gastrostomy: indications, limitations, techniques, and results. World J Surg. 1989;13(2):165-70.

14. MacLean AA, Miller G, Bamboat ZM, et al. Abdominal wall necrotizing fasciitis from dislodged percutaneous endoscopic gastrostomy tubes: a case series.Am Surg. 2004;70(9):827-31.

15. Huang SY, Levine MS, Raper SE. Gastrocolic fistula with migration of feeding tube into transverse colon as a complication of percutaneous endoscopic gastrostomy. AJR Am J Roentgenol. 2005;184(3 Suppl):S65-6.

16. Zerizer I, Chan WH, Singer M. Intramural feeding: a complication of percutaneous endoscopic gastrostomy feeding. Eur J Gastroenterol Hepatol. 2005;17(1):131.

17. Ragunath K, Roberts A, Senapati S, et al. Retrograde jejunoduodenal intussusception caused by a migrated percutaneous endoscopic gastrostomy tube. Dig Dis Sci. 2004;49(11-12):1815-7.

18. Dennis MS, Lewis SC, Warlow C. Effect of timing and method of enteral tube feeding for dysphagic stroke patients (FOOD): a multicentre randomised controlled trial. Lancet. 2005; 365(9461):764-72.

19. Gauderer MW, Ponsky JL, Izant RJ. Gastrostomy without laparotomy: a percutaneous endoscopic technique. J Pediatr Surg. 1980;15(6):872-5.

Endereço para Correspondência:

Dr. Ricardo Zorrón

Av. Ayrton Senna, 1850 s/418

Barra da Tijuca - Rio de Janeiro - Brasil

CEP: 22 775-000

Email: rzorron@terra.com.br 